\title{
LÜDERS THEOREM FOR UNSHARP QUANTUM MEASUREMENTS
}

\author{
PAUL BUSCH AND JAVED SINGH \\ FORMER AFFILIATION (PB): DEPARTMENT OF MATHEMATICS, UNIVERSITY OF HULL, UK \\ CURRENT (PB): DEPARTMENT OF MATHEMATICS, UNIVERSITY OF YORK, UK \\ E-MAIL ADDRESS: PAUL.BUSCH@YORK. AC.UK \\ PUBLISHED IN: PHYSICS LETTERS A 249 10-12 (1998). \\ DOI: 10.1016/S0375-9601(98)00704-X
}

\begin{abstract}
A theorem of Lüders states that an ideal measurement of a sharp discrete observable does not alter the statistics of another sharp observable if, and only if, the two observables commute. It will be shown that this statement extends to certain pairs of unsharp observables. Implications for local relativistic quantum theory will be discussed.
\end{abstract}

\section{INTRODUCTION}

Let $\mathcal{H}$ be a complex separable Hilbert space, $A$ a self-adjoint operator with discrete spectrum and spectral decomposition $A=\sum_{i} a_{i} E_{i}^{A}$. Here the $a_{i}, i=1,2, \cdots, N \leq \infty$, are the distinct eigenvalues, with spectral projections $E_{i}^{A}$ adding up to unity, $\sum_{i} E_{i}^{A}=I$. A non-selective ideal measurement of the observable represented by $A$ gives rise to the Lüders transformation of states (density operators) [1]

$$
\rho \mapsto \mathcal{I}_{L}(\rho)=\sum_{i=1}^{N} E_{i}^{A} \rho E_{i}^{A} .
$$

Let $B$ be any self-adjoint operator on $\mathcal{H}$. Then the Lüders theorem asserts that

$$
\operatorname{tr}\left[\mathcal{I}_{L}(\rho) \cdot B\right]=\operatorname{tr}[\rho \cdot B]
$$

holds for all states $\rho$ exactly when $B$ commutes with all $E_{i}$ and thus with $A$. This result is interpreted as follows: if $A$ and $B$ commute, then the outcomes of a measurement of an observable represented by $B$ do not depend on whether $A$ has been measured first.

In this paper the question will be investigated as to what extent the Lüders theorem pertains also to unsharp observables, represented by POV measures that are not PV measures. If the observable measured first, $A$, is unsharp, it will be represented by a complete set of coexistent effects $E_{i}, i=1, \cdots, N$, 
$\sum_{i} E_{i}=I$. In this case the Lüders transformation is defined as [2]

$$
\rho \mapsto \mathcal{I}_{L}(\rho)=\sum_{i=1}^{N} E_{i}^{1 / 2} \rho E_{i}^{1 / 2} .
$$

The second observable could also be an unsharp observable. In this case, $B$ will represent an effect in the range of the corresponding POV measure. The question is whether commutativity is still necessary for (1.2) to hold for all states.

\section{Generalised Lüders Theorem}

We will formulate and prove two propositions which cover two classes of cases of a general Lüders theorem yet to be proved for arbitrary POV measures.

Proposition 1. Let $E_{i}, i=1,2, \cdots, N \leq \infty$, be a complete family of effects. Let $B=\sum_{k} b_{k} P_{k}$ be an effect with discrete spectrum given by the strictly decreasing sequence of eigenvalues $b_{k} \in[0,1]$ and spectral projections $P_{k}$, $k=1, \cdots, K \leq \infty$. Then $\operatorname{tr}\left[\mathcal{I}_{L}(\rho) \cdot B\right]=\operatorname{tr}[\rho \cdot B][E q$. (1.2)] holds for all states $\rho$ exactly when all $E_{i}$ commute with $B$.

Proof. Commutativity is obviously sufficient for (1.2) to hold. To prove the converse implication, assume that (1.2) holds for all states $\rho$. This is equivalent to the following equation :

$$
B=\sum_{i=1}^{N} E_{i}^{1 / 2} B E_{i}^{1 / 2} .
$$

Let $\varphi \in \mathcal{H}$ be an arbitrary vector. Then Eq. (2.1) gives:

$$
\begin{aligned}
\left\langle P_{1} \varphi \mid B P_{1} \varphi\right\rangle & =b_{1}\left\langle P_{1} \varphi \mid P_{1} \varphi\right\rangle=\sum_{i=1}^{N}\left\langle E_{i}^{1 / 2} P_{1} \varphi \mid B E_{i}^{1 / 2} P_{1} \varphi\right\rangle \\
& \leq b_{1} \sum_{i=1}^{N}\left\langle E_{i}^{1 / 2} P_{1} \varphi \mid E_{i}^{1 / 2} P_{1} \varphi\right\rangle=b_{1}\left\langle P_{1} \varphi \mid P_{1} \varphi\right\rangle .
\end{aligned}
$$

It follows that equality must hold and thus all terms of the first sum must equal the corresponding terms of the second sum. Taking into account the fact that $b_{1}=\|B\|$, this can be expressed as follows: $\left\|\left(b_{1} I-B\right)^{1 / 2} E_{i}^{1 / 2} P_{1} \varphi\right\|=$ 0 , which is to hold for all $\varphi$. Therefore, $B E_{i}^{1 / 2} P_{1}=b_{1} E_{i}^{1 / 2} P_{1}$, and so $P_{1} E_{i}^{1 / 2} P_{1}=E_{i}^{1 / 2} P_{1}$. This implies that all $E_{i}$ commute with $P_{1}$.

Now proceed as follows: substitute $B_{1}=B$ with $B_{2}=B-b_{1} P_{1}$. The commutativity just proven together with Eq. (2.1) entail the same equation for $B_{2}$ :

$$
B_{2}=\sum_{i=1}^{N} E_{i}^{1 / 2} B_{2} E_{i}^{1 / 2}
$$


Applying the same argument as before with $b_{2}=\left\|B_{2}\right\|$ yields the commutativity of all $E_{i}$ with $P_{2}$. Thus one concludes inductively that the $E_{i}$ commute with all $P_{k}$ and hence with $B$.

Proposition 2. Let $E_{1}=E$ be an effect, $E_{2}=I-E$. Let $B$ be any effect. Then $\operatorname{tr}\left[\mathcal{I}_{L}(\rho) \cdot B\right]=\operatorname{tr}[\rho \cdot B][E q$. (1.2)] holds for all states $\rho$ exactly when $E_{1}$ commutes with $B$.

Proof. Eq. (1.2), taken for all states $\rho$, is equivalent to Eq. (2.1) [with $N=2$ ]. If $E_{1}$ commutes with $B$, then (2.1) follows trivially. Conversely, assume this equation holds. By a simple algebraic manipulation one deduces the following:

$$
E_{1} B+B E_{1}=2 E_{1}^{1 / 2} B E_{1}^{1 / 2},
$$

and this is equivalent to

$$
\left[E_{1}^{1 / 2},\left[E_{1}^{1 / 2}, B\right]\right]=0
$$

where $[A, B]$ denotes the commutator of the bounded operators $A, B$. It follows that the self-adjoint operator $C:=i\left[E_{1}^{1 / 2}, B\right]$ is quasi-nilpotent, i.e. its spectrum is $\{0\}$. [This follows from a Lemma stated and proved in the Appendix.] Therefore $C=0$, and thus $E_{1}$ and $B$ commute.

\section{Discussion}

We have generalised the Lüders theorem to two classes of unsharp measurements: in the first case, arbitrary Lüders transformations are allowed while the test effect $B$ has discrete spectrum; in the second case, the spectrum of the effect $B$ is arbitrary but the class of Lüders transformations is restricted to those corresponding to simple observables [i.e. POV measures with ranges $\left.\left\{E_{1}, I-E_{1}\right\}\right]$. Note that Proposition 2 holds not only for effects $B$ but for any bounded self-adjoint operator.

We take these results as indications that the statement of the Lüders theorem can be expected to hold in full generality. Unfortunately, the above simple proofs do not extend in an obvious way to the general case so that further investigations are required. From a physical point of view, however, the present results may already be considered sufficient for the following considerations. If an ideal measurement of an observable defined by the complete set of effects $E_{1}, E_{2}, \cdots$ is realisable, then it seems plausible that it should also be possible to perform ideal measurements of the simple observables $E_{i}, I-E_{i}$. Thus Proposition 2 would apply to each of those.

Now consider the well-known application of the Lüders theorem in the context of relativistic quantum theory. Here the observable $A$ defined by the effects $E_{1}, E_{2}, \cdots$ is taken to belong to a local algebra associated with some bounded spacetime region $X$, and the effect $B$ is considered to belong to a local algebra associated with another bounded region $Y$ with a spacelike separation from the first region. The requirement of (Einstein) causality 
then states that a measurement of any observable $A$ in region $X$ should not have an observable effect in region $Y$. This condition is formalised by means of equations (1.2) or (2.1) if the measurement of $A$ is assumed to be an ideal measurement with the ensuing non-selective Lüders transformation (1.11.3). If $A$ is a simple observable, then Proposition 2 applies and we conclude that every effect in the algebra associated with region $Y$ commutes with the effects in the range of $A$. Hence, local commutativity follows from Einstein causality under the weaker assumption of the observables being defined as POV measures rather than self-adjoint operators. This generalises a result of a (fundamental but apparently not too well known) paper by Schlieder [3].

\section{Appendix A. A Lemma}

We present a proof of the following fact: Let $d: \mathcal{A} \rightarrow \mathcal{A}$ be a bounded derivation on the unital $C^{*}$-algebra $\mathcal{A}$ with unit $I$. Let $a \in \mathcal{A}$ be such that $d^{2} a=0$. Then $d a$ is quasi-nilpotent.

First note that $d^{2} a=0$ implies $d^{k} a=0$ for $k=2,3, \cdots$. This can be used to prove by induction that $d^{n}\left(a^{n}\right)=n !(d a)^{n}, n=1,2, \cdots$. It then follows that

$$
\left\|(d a)^{n}\right\|^{1 / n}=(n !)^{-1 / n}\left\|d^{n}\left(a^{n}\right)\right\|^{1 / n} \leq(n !)^{-1 / n}\|d\|\|a\| .
$$

Therefore the spectral radius of $d a, r(d a)$, is

$$
r(d a)=\lim _{n \rightarrow \infty}\left\|(d a)^{n}\right\|^{1 / n}=0,
$$

so that the spectrum of $d a$ is $\{0\}$.

The proof of Proposition 2 makes use of the fact that $d: B \rightarrow\left[E_{1}^{1 / 2}, B\right]$ is a bounded derivation on the algebra of bounded operators.

\section{REFERENCES}

[1] G. Lüders: Über die Zustandsänderung durch den Messprozess. Ann. Physik (6.F.) 8 (1951) 322-328.

[2] P. Busch, P. Lahti, P. Mittelstaedt: The Quantum Theory of Measurement, Springer, Berlin, 2nd revised ed., 1996.

[3] S. Schlieder: Einige Bemerkungen zur Zustandsänderung von relativistischen quantenmechanischen Systemen durch Messungen und zur Lokalitätsforderung. Commun. math. Phys. 7 (1968) 305-331. 